\title{
Gel Formation in Suspensions of Oppositely Charged Colloids: Mechanism and Relation to the Equilibrium Phase Diagram
}

\author{
Eduardo Sanz, ${ }^{* \dagger} \dagger$ Mirjam E. Leunissen, ${ }^{\ddagger}$ Andrea Fortini, ${ }^{\S}$ Alfons van Blaaderen, and \\ Marjolein Dijkstra* \\ Soft Condensed Matter, Debye Institute for NanoMaterials Science, Utrecht University, Princetonplein 5, \\ 3584 CC Utrecht, The Netherlands
}

Received: February 18, 2008; Revised Manuscript Received: May 28, 2008

\begin{abstract}
We study gel formation in a mixture of equally-sized oppositely charged colloids both experimentally and by means of computer simulations. Both the experiments and the simulations show that the mechanism by which a gel is formed from a dilute, homogeneous suspension is an interrupted gas-liquid phase separation. Furthermore, we use Brownian dynamics simulations to study the relation between gel formation and the equilibrium phase diagram. We find that, regardless of the interaction range, an interrupted liquid-gas phase separation is observed as the system is quenched into a state point where the gas-liquid separation is metastable. The structure of the gel formed in our experiments compares well with that of a simulated gel, indicating that gravity has only a minor influence on the local structure of this type of gel. This is supported by the experimental evidence that gels squeezed or stretched by gravity have similar structures, as well as by the fact that gels do not collapse as readily as in the case of colloid-polymer mixtures. Finally, we check whether or not crystallites are formed in the gel branches; we find crystalline domains for the longer ranged interactions and for moderate quenches to the metastable gas-liquid spinodal regime.
\end{abstract}

\section{Introduction}

There has always been great interest in understanding the properties of gels and the gelation process. This is not only because of the large number of potential applications, but also because it poses a fundamental research challenge in understanding systems that are (falling) out of equilibrium. Looking at the nature of the interparticle bonds, gels can be classified as chemical, if the constituents are linked by covalent bonds, or as physical, if the bonds are reversible. Note, however, that in some cases physical gels can effectively become chemical gels, when the bond strength is many times the thermal energy $\left(k_{\mathrm{B}} T\right)$ and the effect of thermal excitations and reversibility become negligible. Here, we study physical gels of colloidal particles with reversible bonds of electrostatic nature. We consider such particle-based suspensions "gelled" when there is at least one non-compact cluster present, which spans space for a time long enough to be detected on an experimentally relevant time scale.

Past and present investigations have been concerned with both the structural and dynamical properties of gels and gelation. In the eighties, there was a resurgence of work on the aggregation and gelation of particle suspensions, in which the concept of fractal self-similarity played an important role (see, e.g., refs 1 and 2 and references cited in ref 3 ). A popular (computer simulation) model from that time is that of diffusion-limited aggregation, ${ }^{4}$ in which monomers diffuse until they stick to a stationary aggregate. In a more realistic version, termed diffusion-limited cluster aggregation (DLCA), diffusion and merger

\footnotetext{
* Corresponding authors. Electronic address: (E.S.) esanz@ph.ed.ac.uk; (M.D.) m.dijkstra1@uu.nl.

$\dagger$ Present address: SUPA, School of Physics, University of Edinburgh, James Clerk Maxwell Building, Kings Buildings, Edinburgh EH9 3JZ, UK.

$¥$ Present address: Center for Soft Matter Research, Physics Department, New York University, 4 Washington Place, New York, NY 10003.

$\S$ Present address: Department of Physics, Yeshiva University, 500 West 185th Street, New York, NY 10033.
}

of the aggregates is also included, while reaction-limited aggregation takes reversibility of the bonds into account. The implication of these early, relatively simple models for colloid science has already been reviewed on several occasions. ${ }^{1,2}$

More recently, there has been a renewed interest in gels, with emphasis in understanding gelation based on the phase behaviour that would be present if the system did not fall out of equilibrium. For instance, from experiments and computer simulations, there are strong indications that gelation can be due to dynamical arrest during a spinodal decomposition. ${ }^{5-8}$ In this case, the particles in the dense phase connect up and become arrested if their mutual attraction is sufficiently strong. Note, however, that there are also cases in which the arrested dense phase locally crystallizes. ${ }^{9}$ Gels can also form outside the borders of the gas-liquid phase separation. For instance, this is found for an interaction potential that restricts the number of bonds that each particle can form. ${ }^{10,11}$ (See ref 12 for a comprehensive review of the various routes toward colloidal gelation). One approach to understanding out-of-equilibrium gelation based on the underlying equilibrium behaviour involves the extension of mode coupling theory (MCT) to dilute suspensions. ${ }^{13,14}$ MCT was developed to understand the glass transition, using equilibrium structural information to describe dynamical arrest, and has been successfully used to predict arrested states in concentrated suspensions. ${ }^{15}$

Here, we use computer simulations and experiments on colloidal suspensions to acquire more insight into the mechanism of gel formation and the relation with the equilibrium phase diagram. A big advantage of colloidal systems is the possibility to visualize gels on a single particle level in 3D real space. ${ }^{16-19}$ Such real-space analysis of gels is important, because it provides us with local structural and dynamical information that is hard to obtain from scattering experiments. For instance, it has been shown that the peak in the structure factor that is often seen in scattering experiments on gelating systems can have quite 
different structural and mechanistic origins. ${ }^{3}$ These differences can be investigated in more detail with real-space experiments and computer simulations. ${ }^{6,20,21}$

In most of the gel-forming colloidal suspensions studied so far, the interactions between the particles were purely attractive and short-ranged (as compared to the particle size), 5,7,8,18,22 although in some cases they were combined with long-ranged repulsions. ${ }^{17}$ There are only a few reports on the aggregation and gelation of binary suspensions with repulsions between similar particle species and attractions between dissimilar species, of which suspensions with oppositely charged particles are the most common. ${ }^{24-26}$ In all the experimental studies, the contact energy was very high, thus nearing the limit of chemical gels (see above). Quite recently, however, a system of micronsized oppositely charged particles was realized with a particle charge small enough to give rise to equilibrium phase behaviour (binary crystal formation). ${ }^{27-29}$ As we noticed that gels can form already when the interactions are still close to giving equilibrium behaviour and as we are not aware of any other experimental studies under such near-equilibrium conditions, we here investigate this type of gel formation both experimentally and with computer simulations.

In our experimental system, we can set the range of the (electrostatic) interactions through the salt concentration in the suspending liquid. For high salt concentrations, there is a metastable gas - fluid coexistence, similar to that of short-ranged attractive systems. ${ }^{30}$ It is therefore of interest to investigate whether the gelation process can be seen as an arrested spinodal decomposition, just like in certain systems with depletioninduced attractions, which are susceptible to thermal fluctuations. ${ }^{7,8}$ For this, we visualized the gelation process by confocal microscopy and compared our experimental observations with computer simulations of a regular spinodal decomposition and of a spinodal decomposition that evolves into an arrested network. We also used computer simulations to study the dependence of the gelation process on the range of the interaction potential, as we do not have full experimental control yet over the exact interaction parameters. The combination of experiments and simulations is also beneficial in other respects: by performing the (Brownian dynamics (BD)) simulations without gravity and without hydrodynamic interactions between the particles and comparing them with the experimental observations, we can draw tentative conclusions about the importance of these factors for the final structure. We also checked whether crystallization took place in gel-like samples by analyzing the configurations obtained both in the experiments and in the simulations.

\section{Technical Details}

2.1. Experiment. 2.1.1. Particle Synthesis. We used two different batches of poly(methyl methacrylate) (PMMA, density $\rho=1.19 \mathrm{~g} / \mathrm{mL}$ ) particles, which were covalently labeled with the fluorescent dyes rhodamine isothiocyanate (RITC) and 7-nitrobenzo-2-oxa-1,3-diazol (NBD), and sterically stabilized with poly-12-hydroxystearic acid. We synthesized these particles by means of dispersion polymerization (for details, see ref 31 ). Before the covalent attachment of the stabilizer to the particle surface (locking), we added a small amount of additional stabilizer (about half of what was used in the synthesis) to the RITC-labeled particles, so as to obtain a larger difference in surface charge between the RITC- and NBD-labeled particles. After the locking step, we washed the particles extensively with hexane and petether to remove any unreacted species and excess stabilizer. The resulting RITC-labeled particles had a diameter of $2.00 \mu \mathrm{m}$, and the NBD-labeled particles were $2.01 \mu \mathrm{m}$. For both particle batches the size polydispersity was $3 \%$, as determined from static light scattering measurements.

2.1.2. Suspensions. We dispersed the particles in a mixture of as-received cyclohexylbromide (CHB, $\rho=1.33 \mathrm{~g} / \mathrm{mL}$, Fluka) and $27.2 \%$ cis-decalin ( $\rho=0.89 \mathrm{~g} / \mathrm{mL}$, Sigma-Aldrich) by weight. This mixture nearly matched the refractive index of the particles, but had a small density mismatch of approximately $-0.1 \mathrm{~g} / \mathrm{mL}$, causing slow sedimentation of the particles. By adding $1.0 \mu \mathrm{M}$ tetrabutylammonium bromide salt (TBAB, Sigma-Aldrich), we tuned the charges of the NBD-labeled and RITC-labeled species such that they were of opposite sign. ${ }^{27,32}$ We found this salt concentration, at which one of the species reverses its charge, by applying an external electric field between two thin wires (T2 thermocouple alloy wire, $50 \mathrm{~m}$ diameter, Goodfellow) threaded through the microscopy sample cell and watching the particle direction of electrophoretic motion. The salt solution was prepared by saturating CHB with TBAB $(\sim 260$ $\mu \mathrm{M}$ ), adding $27.2 \mathrm{wt} \%$ cis-decalin and then diluting this with a salt-free $\mathrm{CHB}$-decalin mixture to the desired TBAB concentration. For the final suspensions, we let separate suspensions of the individual particle species equilibrate overnight before mixing them together; after this, the behavior of the plus-minus mixture remained unchanged for more than a year. We used suspensions with an overall weighed-in volume fraction of $\phi$ $=0.05$.

On the basis of conductivity (Scientifica 627 conductivity meter) and electrophoretic mobility (Coulter Delsa 440 SX) measurements, we estimated values for the Debye screening length and particle charges of our suspensions (see ref 33 for details). The inverse of the Debye screening length times the particle diameter is between 1 and 4 , and the number of particle charges is between 50 and 300 . We also determined the dielectric constant of the particle-free solvent mixture, $\varepsilon_{\mathrm{S}}=5.6$, through correlation with the measured refractive indexes of several mixtures and the pure CHB and cis-decalin solvents. ${ }^{33}$ The viscosity of our $\mathrm{CHB}$-decalin mixture was $2.217 \mathrm{mPas}$ at $25^{\circ} \mathrm{C}$ (measured with a Schott ViscoSystem).

2.2. Simulations. In our simulations, the screened Coulomb interaction between two colloids of diameter $\sigma$ and charge $\mathrm{Ze}$ is approximated by a Yukawa potential plus a repulsive core:

$$
u(r) / k_{\mathrm{B}} T=\left\{\begin{array}{cc}
u *\left(\left(\frac{\sigma}{r}\right)^{36}+S\right) & r<\sigma \\
\pm u * \frac{\mathrm{e}^{-\kappa(r-\sigma)}}{r / \sigma} & \sigma \leq r<r_{\mathrm{c}} \\
0 & r \geq r_{\mathrm{c}}
\end{array}\right.
$$

where $r$ is the distance between the center of the colloids, $u^{*}$ is the energy at contact in $k_{\mathrm{B}} T$ units, and $r_{\mathrm{c}}$ is the cut-off radius (set at the distance where $u(r) /\left(u^{*} k_{\mathrm{B}} T\right)=10^{-3}$ ), and $S$ is a constant whose value is 0 for the interaction between equally charged particles and -2 between oppositely charged particles. This constant does not affect the force and, hence, it does not enter in the BD simulations. Nevertheless, it should be taken into account if one wishes to compare BD with Monte Carlo simulations. The sign of the interaction potential is positive for equally charged colloids and negative otherwise. The energy in $k_{\mathrm{B}} T$ when $r=\sigma, u^{*}$, is equal to $Z^{2} \lambda_{\mathrm{B}} /\left((1+\kappa \sigma / 2)^{2} \sigma\right)$, where $\lambda_{\mathrm{B}}=e^{2} / \varepsilon_{\mathrm{s}} k_{\mathrm{B}} T$ is the Bjerrum length ( $\varepsilon_{\mathrm{S}}$ is the dielectric constant of the solvent) and $\kappa=\left(8 \pi \lambda_{\mathrm{B}} \rho_{\text {salt }}\right)^{1 / 2}$ is the inverse Debye screening length ( $\rho_{\text {salt }}$ is the number density of added salt ions). The steep repulsive potential $\left(1 / r^{36}\right)$ prevents overlap between the colloids. Figure 1 shows the interparticle potential for the screening lengths studied in our simulations: $\kappa \sigma=2$ and $\kappa \sigma=$ 


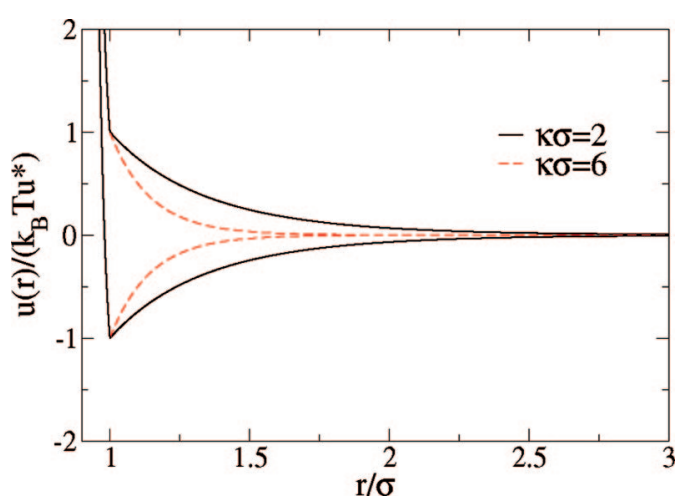

Figure 1. Interaction potential, between equally (positive curves) and oppositely charged particles, as a function of the distance for the two screening lengths under study.
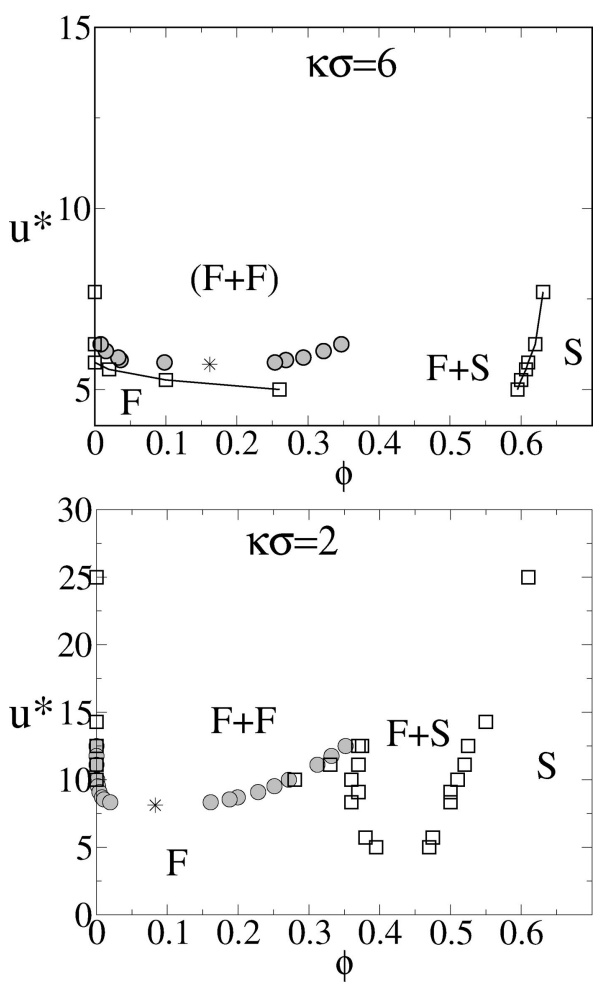

Figure 2. Phase diagram of oppositely charged colloids modeled with screened Coulombic plus hard-core interactions, for two different screening parameters $\kappa \sigma .30$ "F" stands for fluid, " $\mathrm{S}$ " is for solid, " $\mathrm{F}+\mathrm{F}$ " is for gas-liquid coexistence, and " $\mathrm{F}+\mathrm{S}$ " is for fluid-solid coexistence. The fluid-solid coexistence points are indicated by squares, the fluid-fluid coexistence is indicated by circles, and the critical point is indicated by an asterisk. Note that the critical point is stable for $\kappa \sigma=$ 2 , whereas it is metastable for $\kappa \sigma=6$.

6. The phase diagram for a very similar model potential (replacing the $1 / r^{36}$ repulsion by a hard-core potential) reproduces the solid structures that are found experimentally in mixtures of oppositely charged colloids. ${ }^{28}$ In Figure 2, we show the phase diagram in the $u^{*}$ packing fraction $(\phi)$ plane. We search for gel formation in the low packing fraction, high attraction region of the equilibrium phase diagram. Although $\kappa \sigma=6$ is outside the experimental range of the present study $(\kappa \sigma \in 1-4)$, it is interesting to study gel formation for this case as well, because the phase diagram for this screening length shows a qualitative difference compared to the phase diagram for $\kappa \sigma=2$ : the critical point is metastable.

We have carried out BD simulations at constant number of particles, volume, and interaction strength $\left(N, V, u^{*}\right)$. Starting from an equilibrated, homogeneous fluid at low $u^{*}$, we quenched the system at higher $u^{*}$ and let it evolve. In this type of simulation, a particle displaces as a result of the force field created by the surrounding particles and because of the collisions and the friction with the "effective" solvent. This means that hydrodynamic interactions are neglected, but we do not expect that this changes the scenario qualitatively. ${ }^{34}$ This expectation is supported by the experiments, where solvent flow did not substantially affect the mechanism of gel formation (see below). The equation of motion is a simple first-order differential equation that can be solved with an Euler integration algorithm:

$$
\alpha_{i}(t+\Delta t)=\alpha_{i}(t)-\frac{\Delta t}{\zeta} \sum_{j \neq i}\left[\frac{\partial u(r)}{\partial r}\right]_{r=r_{i j}} \frac{\alpha_{i j}}{r_{i j}}+\delta \alpha_{i}\left(u^{*}, \Delta t\right)
$$

Here, $\alpha_{i}$ represents a coordinate $(x, y$, or $z)$ of the $i$ th particle, $\Delta t$ is the time step, $\zeta$ is the friction coefficient (which is related to the self-diffusion constant $D_{0}$ and the solvent viscosity $\eta$ by the Stokes-Einstein relation: $\left.\zeta=k_{\mathrm{B}} T / D_{0}=3 \pi \eta \sigma\right), r_{i j}$ is the distance between particles $i$ and $j, \alpha_{i j}$ is the distance along the $\alpha$ coordinate, and $\delta \alpha_{i}\left(u^{*}, \Delta t\right)$ is the displacement due to collisions with the solvent, which is a random number extracted from a Gaussian distribution with 0 mean and variance $2 D_{0} \Delta t$. In our simulations, the unit of length is the particle diameter $\sigma$, and the unit of energy is the contact energy $u^{*}$. For colloidal systems, where the control parameter is not the temperature but the strength of interaction, it is more sensible to use $u^{*}$ rather than $k_{\mathrm{B}} T$ as the energy unit. Consistently with our choice of length and energy units, the unit of time in the simulations is $t^{*}=\sigma^{2} /\left(D_{0} u^{*}\right)$ and the variance is equal to $2 \Delta t^{*} / u^{*}$. We have chosen $\Delta t$ to be equal to $7 \times 10^{-6} t^{*}$. If $\Delta t$ is too small, the simulations become too expensive. On the other hand, if it is too large, the particles would unrealistically overlap, causing large repulsive forces that would not be present in a small $\Delta t$ simulation. We are confident to have properly chosen $\Delta t$ because we obtain the same average internal energy both with a Monte Carlo and with a BD simulation (for an equilibrium state). Given the viscosity of the solvent $(2.217 \mathrm{mPas})$, we use the Stokes-Einstein relation to estimate the self-diffusion coefficient, $D_{0} \approx 10^{-13} \mathrm{~m}^{2} / \mathrm{s}$. Hence, one $\sigma^{2} /\left(D_{0}\right)$ time unit roughly corresponds to $40 \mathrm{~s}$ in the experiments (if we neglect the volume fraction dependence of hydrodynamic interactions).

\section{Results and Discussion}

To study experimentally the gel formation process from the very first onset of aggregation, we glued a $0.1 \times 1.0 \mathrm{~mm}$ glass capillary (VitroCom) onto a microscopy slide and mounted this on the stage of a confocal scanning laser microscope (Leica SP2 CSLM), focusing inside the sample space. We then prepared a homogeneous sample by severely shaking the stock vial with a suspension of the two particle species, thus disrupting any gel-like structures that had formed already, and letting the capillary forces suck the suspension into the sample space. Immediately after, we started the confocal imaging, without sealing the sample cell.

Figure 3 a shows a sequence of pictures of a $100 \times 100$ $\mu \mathrm{m}^{2}$ area of the sample illustrating the gel formation process starting from a dilute colloidal fluid. The colloidal packing fraction of the initial suspension was 0.05 . Initially, colloids aggregated into chains with thickness on the order of a single particle. Subsequently, the chains coarsened, forming a percolating network in which there was still motion of particles and aggregates. Finally, the network branches coarsened further by coalescence with neighbouring branches, 
(a)
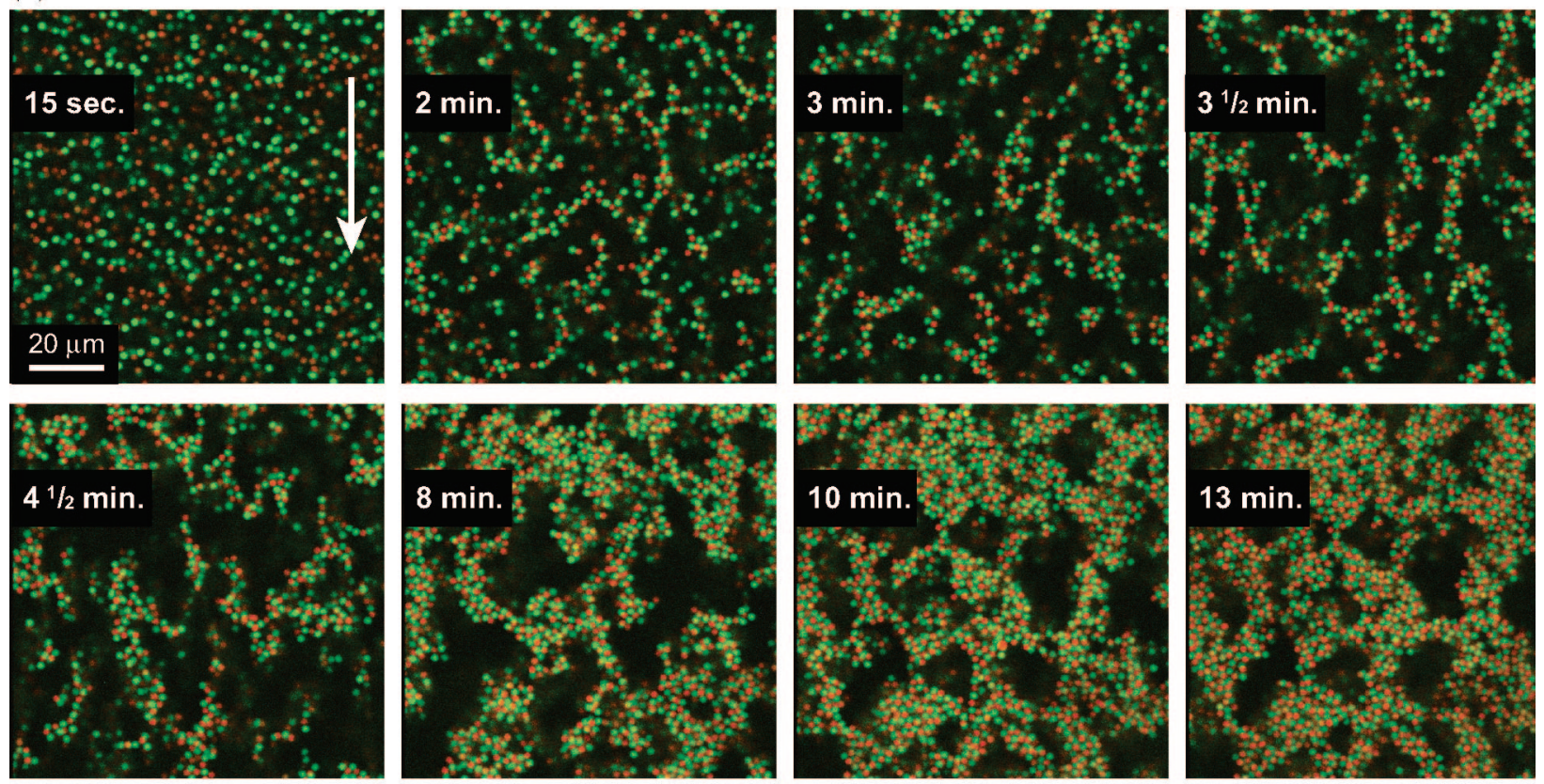

(b)
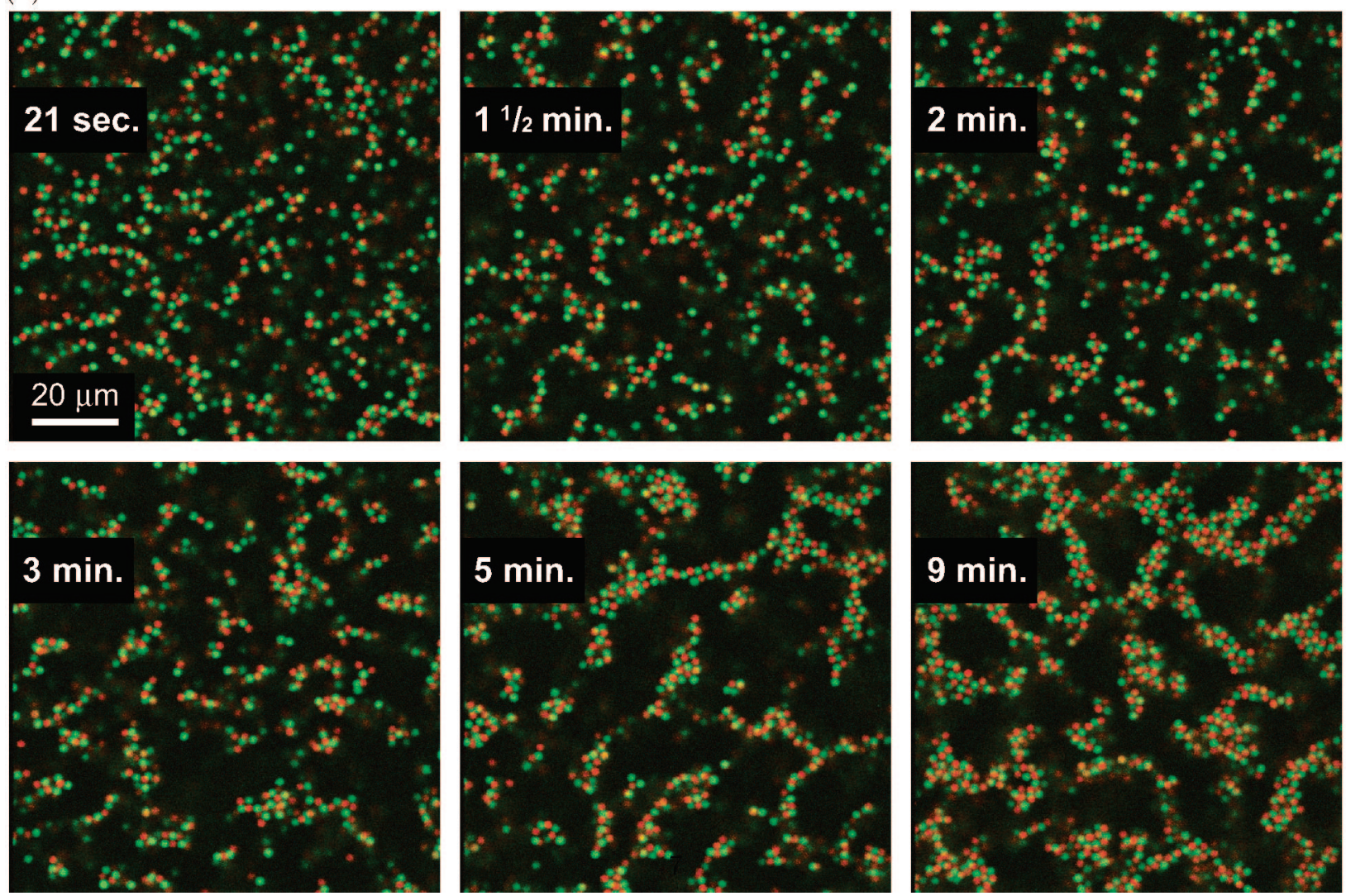

Figure 3. Sequence of confocal snapshots illustrating the formation of a gel with solvent flow (a) and without solvent flow (b) (flow direction given by the arrow). Red particles are positively charged, and green particles are negatively charged. The elapsed time is indicated in the frames. Movies illustrating the whole sequence are provided as Supporting Information.

and an arrested gel was formed. In this particular experiment, there was a solvent flow along the length of the capillary due to its open ends. The presence of the solvent flux allowed us to confirm that the structure formed was indeed a gel with a yield stress, given that it was able to bear the drag exerted by the flowing solvent. Note that the last two pictures of the sequence are quite similar, indicating that, beyond a certain point, the structure of the colloidal network was not affected by the solvent flow. Because of the small density mismatch between the particles and the solvent the gel network occupied only the bottom half of the capillary (see experimental details). The packing fraction of the sediment was $\sim 0.1$. We confirmed that the solvent was still flowing even after the evolution of the structure stopped by focusing the 
microscope on the top part of the capillary, where free colloids were still being dragged along by the solvent.

In order to check that the flow did not qualitatively affect the gel formation mechanism described above, we repeated the experiment, sealing the ends of the capillary immediately after introducing the sample. Figure $3 \mathrm{~b}$ gives an overview of the evolution of the system in this case. Apparently, the qualitative picture was not altered. As it can be expected though, the initially formed colloidal chains were isotropically oriented, in contrast with some alignment along the flow direction observed in the non-sealed capillary.

In order to check whether the experimental observations can be interpreted as an arrested spinodal decomposition, it would be useful to know the exact state point at which the experiments have been carried out in the equilibrium phase diagram. Unfortunately, the fact that the number of charges on the colloids is coupled to the screening length ${ }^{27}$ makes it difficult to scan the parameter space and to draw up the full experimental phase diagram. However, the phase diagram of the model presented in the previous section, which predicts the existence of the solid structures observed experimentally, ${ }^{27,28}$ is already known, ${ }^{28,30}$ offering an opportunity to study, by means of computer simulations, gel formation for oppositely charged colloids in the context of an equilibrium phase diagram.

Let us therefore discuss the results obtained with BD simulations. In order to study the formation of gels, we quenched a homogeneous system of 686 colloids (343 positively charged plus 343 negatively charged), equilibrated at a state point where the fluid is the stable phase $(\kappa \sigma=6, \phi$ $=0.1, u^{*}=1$ ), into regions of the phase diagram where there is gas-liquid spinodal decomposition. It is already known, for short-ranged attractive particles, that gel formation can be related to gas-liquid phase separation. .,35,7,8 $^{\text {The same }}$ can be stated for oppositely charged particles after the computer simulation work of Caballero and Puertas, ${ }^{26}$ although the metastability of the gas-liquid coexistence with respect to the gas - solid was not discussed in their work (the complete phase diagram for their model potential has been recently calculated). ${ }^{36}$ Our model potential, however, is different from theirs. Moreover, we do not introduce any polydispersity, and therefore we do not exclude the possibility of crystallization. Additionally, we use BD simulations, as opposed to molecular dynamics, which, qualitatively, are closer to the experiments. We therefore check the regions of the phase diagram in which our model forms gels. In order to check whether particles' motion is arrested, we have calculated the mean square displacement for the structures formed 955 units of time after the quench (Figure 4). Each curve has been calculated by averaging the results over five runs, starting from the same configuration but with a different seed for the random number generator. The results for $\kappa \sigma=$ 6 are shown in Figure 4a. The inflection point in the mean square displacement, characteristic of particle arrest, becomes evident as the depth of the quench into the metastable spinodal decomposition region increases (see phase diagram in Figure 2). For $\kappa \sigma=6$ the interaction range is rather short and, as a consequence, the critical point is metastable. In contrast, for $\kappa \sigma=2$, the range is larger, and the critical point is stable. Nonetheless, beyond the triple point $\left(u^{*}>12.5\right)$, the gas-liquid coexistence becomes metastable. As is shown in Figure $4 b$, there is a clear inflexion point in the mean square displacement if the system is quenched to an energy at contact well above the triple point for $\kappa \sigma=2$. Therefore,
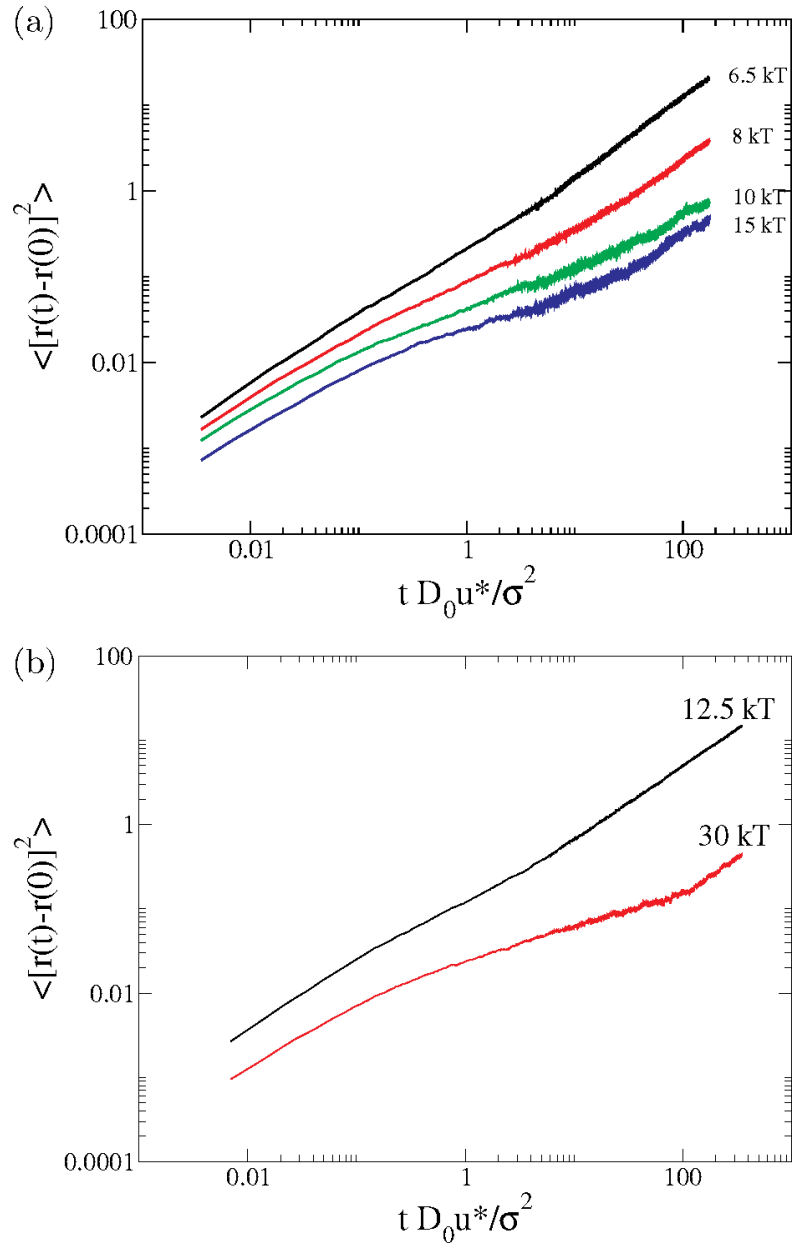

Figure 4. Mean square displacement for the structures formed 955 $\sigma^{2} /\left(D_{0} u^{*}\right)$ after the quench: (a) for $\kappa \sigma=6$ and (b) for $\kappa \sigma=2$. In the figure, the energy at contact corresponding to each curve is indicated.

in order to obtain dynamical arrest in a low packing fraction sample of oppositely charged colloids, it is not mandatory that the range of interaction is so short as to bury the critical point underneath the gas-solid coexistence. The sine qua non requisite for a system to be able to have an interrupted liquid-gas phase separation is that the system is quenched to a state point where the gas-liquid coexistence is metastable with respect to the gas-solid. This can be fulfilled for the whole range of gas-liquid coexistence, if the critical point is metastable (short-range interactions), or only below the triple temperature (above the triple $u^{*}$ ) if the critical point is stable (long-range interactions). The possibility of gel formation in systems interacting with long-ranged attractions (stable critical point) has already been considered by Lodge and Heyes for the Lennard-Jones potential. ${ }^{20}$ Note that, although the metastability of the gas-liquid coexistence is a necessary condition for gelation for the interaction potentials studied here, it is not a sufficient one: the quench must also be deep enough, so that the motion of the particles is sufficiently restricted, thereby resisting the spinodal coarsening. In other words, the liquid formed in the first stages of the spinodal decomposition must turn into a (strong) attractive glass.

Additionally, we can look for signatures of dynamical arrest in the decay of the bond correlation function, $v(t),{ }^{37,38}$ which is the fraction of bonds that remains equal to those present at time zero: 


$$
v(t)=\frac{\sum_{i<j} \theta\left(r_{i j}(0)\right) \theta\left(r_{i j}(t)\right)}{\sum_{i<j} \theta\left(r_{i j}(0)\right)}
$$

$\theta\left(r_{i j}\right)$ is 1 if the distance between particles $i$ and $j, r_{i j}$, is less than 1.3 particle diameters and 0 otherwise. Hence, $v(t)$ will be equal to 1 at time $t=0$ and will decay to small values as particles lose track of their initial neighbours. In Figure 5, $v(t)$ is shown for the same state points at which the mean square displacement has been calculated. Similarly to the mean square displacement, the bond correlation function has been calculated starting from a configuration obtained 995 time units after the quench and averaging over five runs. For $\kappa \sigma=6$, it is evident how the dynamics is increasingly slowed down as the quench into the metastable spinodal decomposition regime became deeper. For the most shallow quench, $u^{*}=6.5$, the correlation function decays to values close to zero within the simulation time. In contrast, for $u^{*}=15, v(t)$ is essentially flat at intermediate times, and it does not decay below 0.85 at the end of the simulation time. For $\kappa \sigma=2$, the situation is similar: $v(t)$ decays rapidly for $u^{*}=12.5$, which corresponds to a state point where the spinodal decomposition is stable, whereas it does not decay below 0.83 for $u^{*}=12.5$. Although it would be nice to establish a line of demarcation for the gel formation, we have seen that the slowing down of the dynamics is gradual. Hence, we are not able to establish a sharp transition to the gel state,
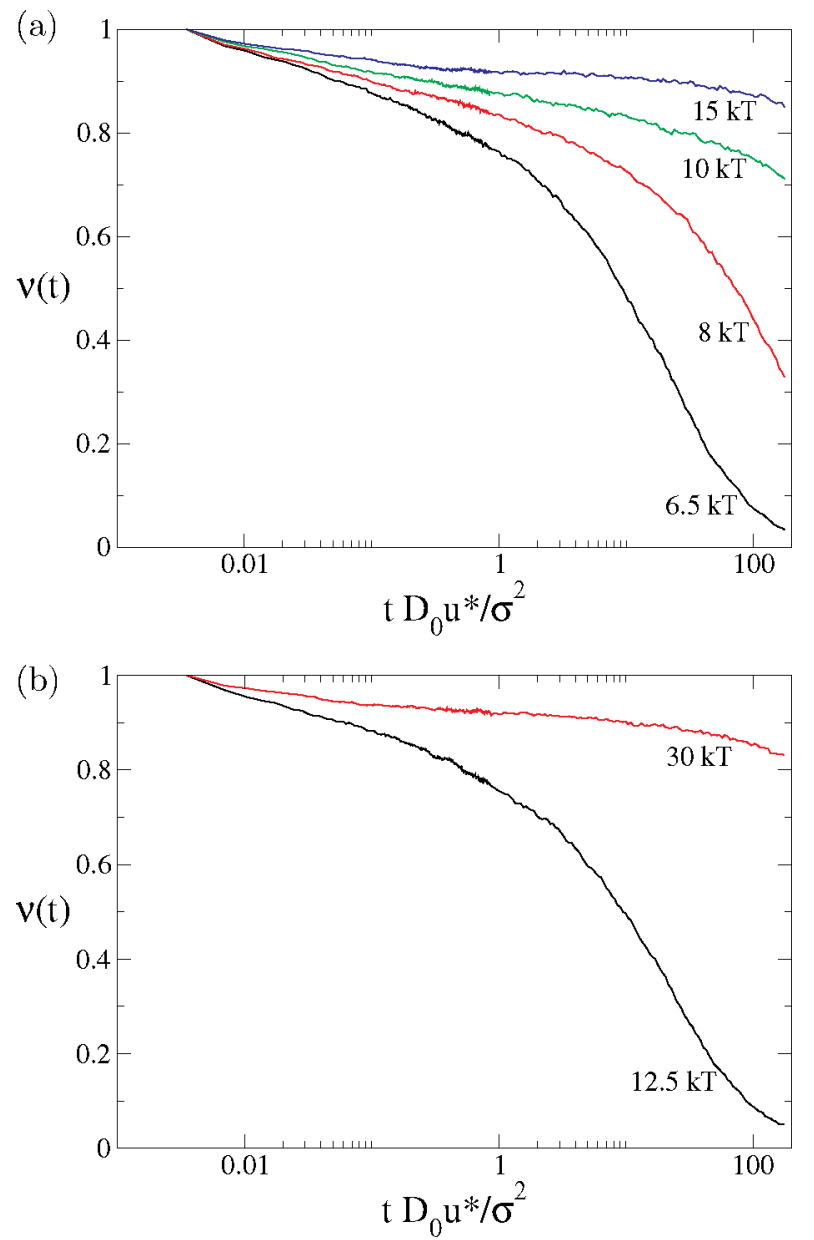

Figure 5. Bond correlation function for structures formed $955 \sigma^{2} /$ $\left(D_{0} u^{*}\right)$ after the quench: (a) for $\kappa \sigma=6$ and (b) for $\kappa \sigma=2$. In the figure, the energy at contact corresponding to each curve is indicated. although it is clear that the deeper the quench into the metastable gas-liquid spinodal, the more evident the arrest signatures become. It would be interesting to check whether, by determining mechanical properties such as the yield stress, it is possible to establish a sharper transition to the gel state, although this is beyond the scope of this work. Nevertheless, the experiment showing a network able to resist the drag force exerted by the solvent, as well as the squeezing by gravity, already proves that oppositely charged particles can form networks of particles with non-zero yield stress. For our present purposes, we arbitrarily define a gel as the structure for which $v(t)$ does not decay below 0.8 after $175 \sigma^{2} /\left(D_{0} u^{*}\right)$. Consequently, we can say that gels have been formed for $u^{*}=15, \kappa \sigma=6$ and $u^{*}=30, \kappa \sigma=2$.

It is interesting to note how the contact energy value that is required to arrest the system varies with the screening length. At $u^{*}=15 k_{\mathrm{B}} T$, for instance, the system is not arrested for $\kappa \sigma$ $=2$, whereas a gel is formed for $\kappa \sigma=6$ at the same contact energy. The position of the point $\left(u^{*}=15, \phi=0.1\right)$ in both equilibrium phase diagrams can help to understand this. For $\kappa \sigma$ $=6, u^{*}=15$ is well above the energy at contact at which the fluid commences to be metastable at packing fraction $0.1\left(u^{*}\right.$ $\approx 5$ ). In contrast, for $\kappa \sigma=2$, a contact energy of $15 k_{\mathrm{B}} T$ is only $3.5 k_{\mathrm{B}} T$ above the triple point $\left(u_{\mathrm{tr}}^{*} \approx 12.5\right)$, where an ergodic liquid can exist. One can also rationalize the different tendencies to arrest by taking a look at the pair potential functions (see Figure 1). Even if the energy at contact is the same for both screening lengths, the change of potential energy with respect to the inter-particle distance is bigger for $\kappa \sigma=6$. Thus, it is easier for a particle to rearrange its position if $\kappa \sigma$ is lower.

The experimental parameters $(\kappa \sigma \in 1-4$, and number of charges $\in 50-300)$ are compatible with a contact energy of tens of $k_{\mathrm{B}} T$, which is a typical value for which we observe gels in our simulations.

For colloid-polymer mixtures with polymer-to-colloid size ratio $q=0.15$, the typical energy at contact beyond which gels are found is $\sim 5 k_{\mathrm{B}} T .{ }^{39}$ This energy is lower than the value of tens of $k_{\mathrm{B}} T$ we find for oppositely charged colloids. The reason is probably that, for colloid-polymer mixtures, the interactions are only attractive, while for oppositely charged colloids, there are both attractive and repulsive interactions between the particles. The equilibrium phase diagram of colloid-polymer mixtures, $q=0.15$, also suggests that the minimum energy at contact required to form a depletion-induced gel is lower than that required to form gels of oppositely charged particles: The contact energy at the metastable gas-liquid critical point is $\sim 3.5$ $k_{\mathrm{B}} T$ and, at the critical volume fraction, the fluid is unstable with respect to the solid beyond an energy at contact of $\sim 2.4$ $k_{\mathrm{B}} T$. These values are higher for the phase diagrams studied here, which is reflected in higher energies at contact required for gel formation.

Let us now compare the mechanism leading to the formation of gels in the simulations with what has been observed in the experiments. The sequence of simulation snapshots of Figure 6 shows the structural evolution from a homogeneous fluid to a percolating arrested network for $\kappa \sigma=6$, at packing fraction 0.1 , and $u^{*}=15$. We know from Figure 4 that particle motion is arrested in the structure eventually formed at this state point. Despite the simplicity of the model (neither gravity nor the effect of hydrodynamic interactions have been taken into account), the qualitative course of gel formation is the same as seen in the experiments: aggregation of particles into a network of thin branches, with single colloidal thickness at the beginning (time, $\left.t=2.3 \sigma^{2} / u^{*} D_{0}\right)$, thickening of the branches $\left(t=12-240 \sigma^{2} /\right.$ $\left.u^{*} D_{0}\right)$, and eventual arrest in a slowly ageing network $(t=$ 


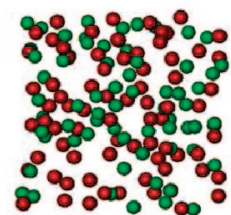

0.12

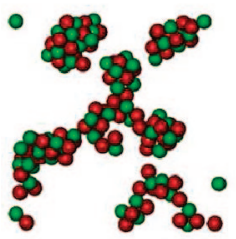

120

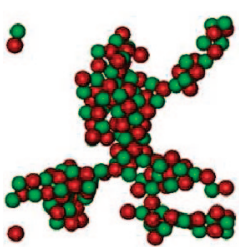

600
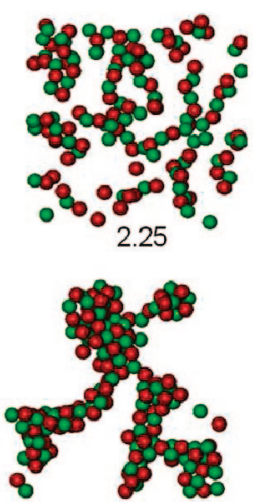

240

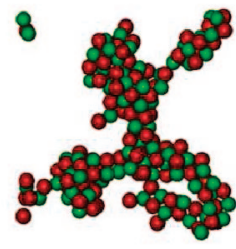

840

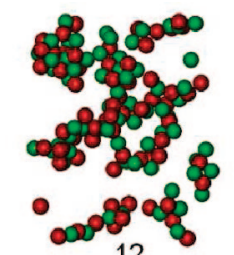

12

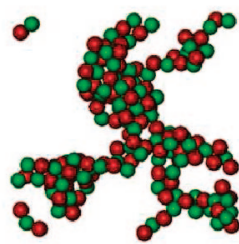

360

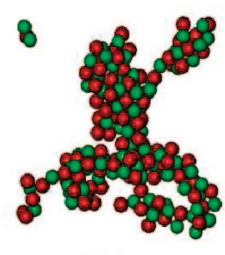

960
Figure 6. Evolution of a homogeneous fluid of oppositely charged Yukawa spheres with $\kappa \sigma=6$ quenched to $u^{*}=15$. The color code is the same as that in Figure 3. For the sake of simplicity and to facilitate a visual comparison with the confocal images of Figure 3, a slice of thickness $3 \sigma$ through the system is shown. The numbers indicate the time elapsed (in $\sigma^{2} /\left(u^{*} D_{0}\right)$ ) after the quench was initiated. A gel phase is eventually formed.

$360-960 \sigma^{2} / u^{*} D_{0}$ ). Note that the network present at 360 , and even at $240 \sigma^{2} / u^{*} D_{0}$, already resembles the final one at 960 $\sigma^{2} / u^{*} D_{0}$. From $t \approx 360 \sigma^{2} / u^{*} D_{0}$ onward, the structure of the network evolves slowly in a process known as aging. ${ }^{40}$ For the other screening length under study, $\kappa \sigma=2$, we observe the same mechanism.

The fact that gels are formed at metastable gas-liquid spinodal state points suggests that the mechanism leading to gel formation might be an arrested spinodal decomposition. In order to check this, we compare the structural evolution of the system when it experiences a (stable) spinodal decomposition, separating eventually into a gas and a liquid, with what happens when the system ends up forming a gel. We compare the aggregation mechanism for quenches to four different state points (all of them at $\phi=0.1$ ): A: $u^{*}=12.5, \kappa \sigma=2$; B: $u^{*}=$ $30, \kappa \sigma=2$; $\mathrm{C}: u^{*}=6.5, \kappa \sigma=6$; D: $u^{*}=15, \kappa \sigma=6$. Point A corresponds to a stable spinodal decomposition, points $\mathrm{B}$ and $\mathrm{D}$ correspond to deep quenches into the metastable spinodal decomposition (eventually forming a gel), and point $\mathrm{C}$ corresponds to a shallow quench to the metastable spinodal. We monitored the number of particles that belong to the biggest cluster, where any two particles which have a distance less than $1.3 \sigma$ belong to the same cluster. Such a cluster is not a compact object but, as we will show from the analysis of $S(q)$, a rather open and branched structure. In Figure 7, the fraction of particles that belong to the biggest cluster $\left(f_{\mathrm{b}}\right)$ is plotted against time (in logarithmic scale). The curves are an average over 20 simulations starting from different homogeneous configurations. At the beginning, for the initial dilute homogeneous system, the fraction is very low, but it increases rapidly as particles form a network-like cluster. It is interesting to note that, in our time units, the fast growth of the cluster occurs at the same time for different state points of a given $\kappa \sigma$, confirming that our time units are convenient to compare the dynamical evolution of the

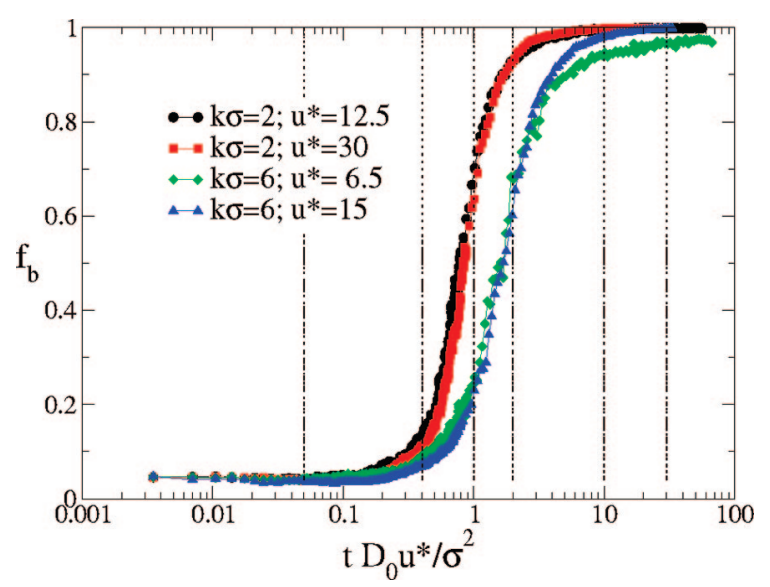

Figure 7. Fraction of particles belonging to the biggest interconnected cluster of the system as a function of time for quenches to different states (see figure legend). The vertical dashed lines indicate the times at which the structure has been analyzed (see main text).
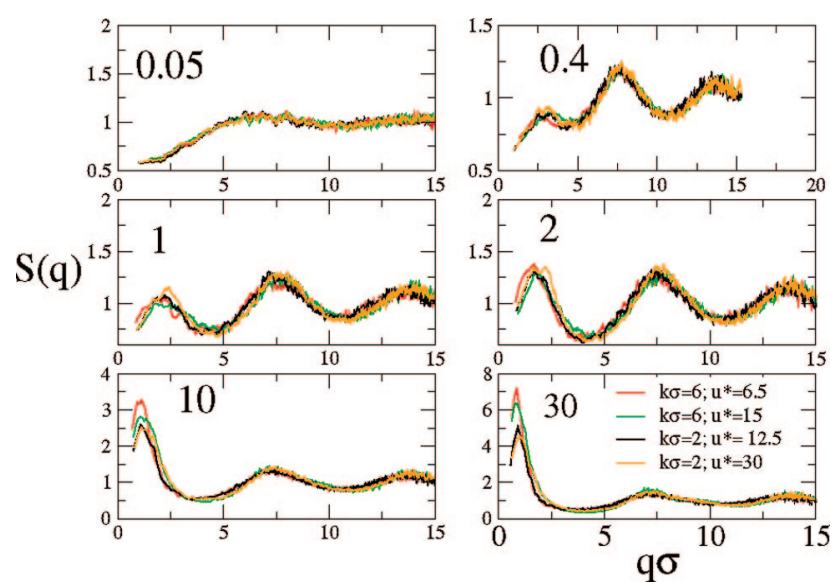

Figure 8. Comparison of the structure factor for different state points at the same time elapsed after the quench (indicated by the numbers inside the graphs (in units of $\sigma^{2} /\left(u^{*} D_{0}\right)$ )).

quenched systems. As can be seen in Figure 7, the longer the interaction range (the lower $\kappa \sigma$ ), the faster the cluster is formed.

According to Figure 7, the fraction of particles belonging to the biggest cluster evolves in a similar fashion for both a spinodal decomposition leading to a gel and for a spinodal decomposition leading to gas-liquid phase separation. We now analyze the structure of the growing cluster at the points indicated by the vertical dashed lines in Figure 7. To do this, we use the structure factor

$$
S(q)=\left\langle\frac{1}{N} \sum_{i=1}^{N} \mathrm{e}^{i \mathbf{q} \mathbf{r}_{i}} \sum_{i=1}^{N} \mathrm{e}^{-i \mathbf{q} \mathbf{r}_{i}}\right\rangle
$$

where the summation is made over all the particles in the simulation box. $\mathbf{q}$ is a vector of the reciprocal space, and $\mathbf{r}_{i}$ is the position of the $i$ th particle. The average is made over $\mathbf{q}$ vectors with the same modulus. In Figure 8 we show, for different times elapsed after the quench, the comparison of $S(q)$ for quenches to state points A, B, C, and D. Each curve is an average over 20 configurations obtained via 20 independent simulations starting form different homogeneous configurations. For $t=0.05 \sigma^{2} /\left(D_{0} u^{*}\right)$, when $f_{\mathrm{b}}$ is small, $S(q)$ is essentially flat, as expected for an unstructured homogeneous fluid. Already when the fraction of particles in the cluster is around 10\% $(t=$ $\left.0.4 \sigma^{2} /\left(D_{0} u^{*}\right)\right)$, a peak at low $q$ starts to develop, indicating the network-like character of the growing cluster. As time passes, 
the low- $q$ peak increases in intensity, and its position shifts towards lower $q$, indicating the formation of a better defined network whose branches thicken. This behavior of $S(q)$ is typical of spinodal decomposition. (Because of the limited size of the simulated system, the low- $q$ peak of the structure factor can at some point no longer be resolved in the coarsening process, but we expect this peak to continue to evolve only very slowly for a quench such as the one shown in Figure 6, beyond 300 time units.) As it was the case for $f_{\mathrm{b}}$, there is no difference in the way $S(q)$ initially evolves, independent of whether the system is quenched to a stable spinodal point or to a metastable gelforming spinodal point. Therefore, our simulations support the idea that the route toward gel formation observed in the experiments is an arrested gas-liquid phase separation. We have not observed, neither in the experiments nor in the simulations, a glass-like transition of particle clusters as a mechanism of gel formation, which is predicted for slow quenches. ${ }^{14,23}$

After 30 units of time have elapsed since the quench, most of the particles belong to a single network (Figure 7), whose signature is the low- $q$ peak in $S(q)$ (Figure 8). To give a clear idea of what the network of particles looks like, we show in Figure 9 a snapshot of such a network for states A, C, and D. The picture is very similar for these three cases, supporting again the idea that the formation of a gel starts in the same way as a spinodal decomposition.

An experimental comparison between a "regular" (fully proceeding) spinodal decomposition and an arrested one (in addition to our computer simulations) would have been illustrative, but unfortunately this proved to be hard to accomplish. For the suspensions used here, we only observed arrested decomposition, likely due to the high particle charge. We found that it is difficult to tune the particle charge and the screening length such that a full decomposition is obtained, ${ }^{30}$ because in our experimental system these parameters are strongly coupled. ${ }^{27}$

Once a gel was formed in our experimental system, we were able to extract the $3 \mathrm{D}$ particle coordinates by scanning the sample with the confocal microscope. In Figure 10a, a snapshot of a gel sedimented on the bottom of the capillary is shown. The fact that gravity is not able to drive the system to a uniform sediment of particles is further proof of the existence of a gel. A snapshot of a gel obtained by simulation is shown in panel $\mathrm{b}$ of the same figure. We can now compare the structure of both gels by means of the radial distribution function $(g(r))$. This comparison is shown in Figure 11. The position of the peaks of the $g(r)$ is similar for both types of interactions (equally and oppositely charged), although, because of the error associated with the position of the particles and with the polydispersity, the experimental peaks of $g(r)$ broaden, becoming significantly lower than in the case of the simulations. Nevertheless, the integration of $g(r)$, which yields the average number of particles at distance $r$ from a central one $(N(r))$, should be comparable. Figure 12 shows that this is indeed the case. Given that the $g(r)$ peaks are sharper in the simulation, the $N(r)$ function for the simulated gels is more "step-like" than for the experimental ones. Nevertheless, the value of $N(r)$ coincides at the end of each step, meaning that the broad peaks of the experimental $g(r)$ contain the same number of particles as the high thin peaks of the $g(r)$ obtained by simulations. From Figure 12 we learn that, in this type of gel, a particle is surrounded by roughly nine others (oppositely charged) in the first coordination shell. According to Figure 12, the structure of the experimental gel resembles the simulated case more closely for $\kappa \sigma=2$. The fact that for $\kappa \sigma=6$ there are less particles than for $\kappa \sigma=2$ beyond (a)

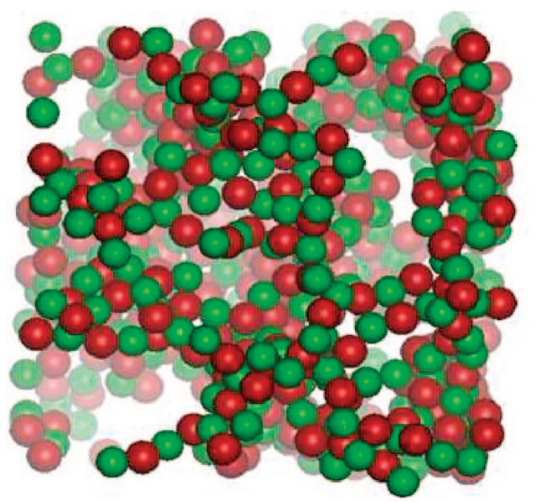

(b)

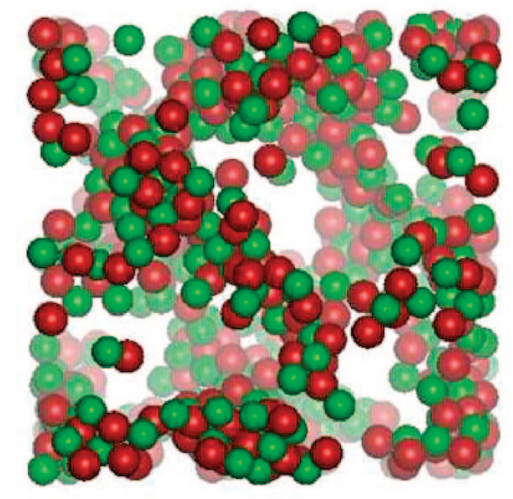

(c)

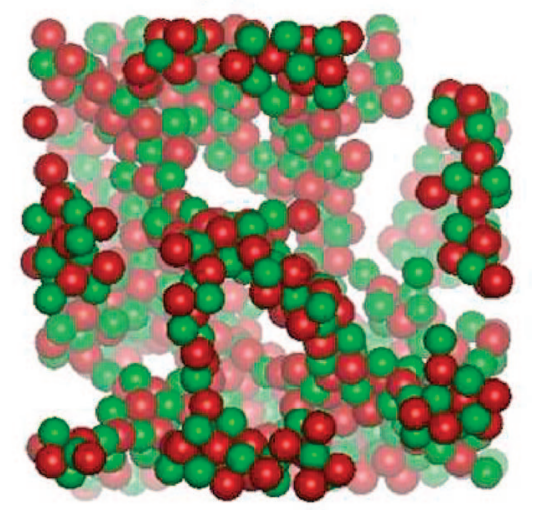

Figure 9. Snapshot of the system $30 \sigma^{2} /\left(D_{0} u^{*}\right)$ after the quench for $\kappa \sigma=2, u^{*}=12.5$ (top); $\kappa \sigma=6, u^{*}=6.5$ (middle); and $\kappa \sigma=6, u^{*}$ $=15$ (bottom). (Color code the same as in Figure 3).

a certain distance is an indication that the gel branches are thinner for $\kappa \sigma=6$.

As a result of electrostatic interactions, some particles got stuck to the walls of the capillary sample cell. As a consequence, we observed in our sample gel-like branches hanging from the top of the capillary, in addition to the sediment on the bottom (see Figure 13 for a snapshot). Interestingly, as Figure 14 illustrates, the $g(r)$ of the gels hanging from the top wall is rather similar to the $g(r)$ of the sedimented gels. Since gravity stretches gels hanging from the top, whereas it squeezes sedimented gels, this is an indication that gravity does not significantly affect the local structure of a gel formed by oppositely charged particles (which was already suggested by the good agreement between the $g(r)$ from the simulations (no gravity) and the $g(r)$ from 
(a)

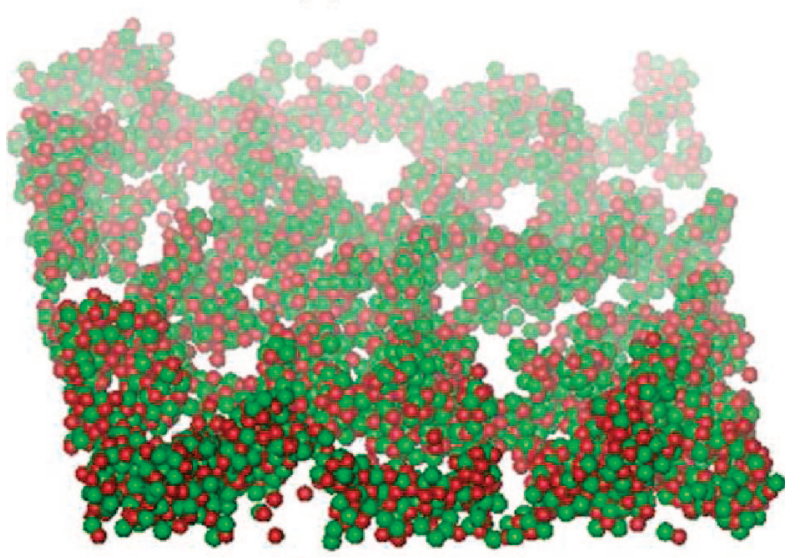

(b)

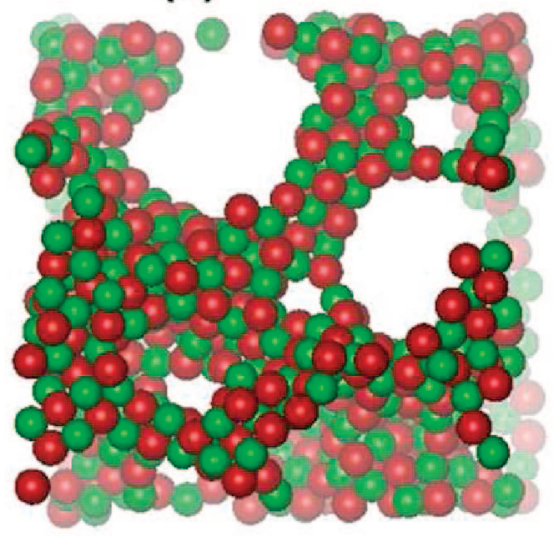

Figure 10. (a) Rendering of the experimentally determined particle coordinates in a gel of oppositely charged PMMA particles. Color code is the same as in Figure 3. The particles were slightly denser than the solvent, which caused sedimentation of the gel on the bottom of the capillary. The packing fraction of the initial homogeneous dispersion was 0.05 . The packing fraction of the gel decreased with the height from the capillary bottom $(h): \phi$ is $\sim 0.2$ in a section between $h=0$ and $3 \sigma ; 0.12$ between $h=3$ and $6 \sigma$, and 0.025 between $h=$ 6 and $9 \sigma$. (b) Snapshot of a gel simulated with $\kappa \sigma=2$ and $u^{*}=30$ $k_{\mathrm{B}} T$, formed $945 \sigma^{2} /\left(D_{0} u^{*}\right)$ after the initial quench.

the experimental gels). Although we have not carried out a systematic analysis of gel collapse, the fact that we can observe for several months both sedimented and "hangingfrom-the-top gel", suggests that this type of gel is much more stable in a gravitational field than gels formed in colloid-polymer mixtures. ${ }^{41}$ Given that gel collapse is an undesirable event for gels used in food or cosmetics, gels of oppositely charged particles have promising properties. In a fairly recent publication, some of us have shown that gravitational effects often lead to dense liquid-like sediments in systems of oppositely charged colloids, ${ }^{27}$ even if they are nearly density matched. Thus, even though the density mismatch is small, gravity can have a non-negligible impact on our system. However, if a gel is formed, the percolating network does not collapse.

Since the simulations were carried out with monodisperse particles and the experimental polydispersity is low (less than $3 \%$ ), we can not rule out the possibility of crystallization in both cases. After all, the crystal $(\mathrm{CsCl})$ is more stable than the liquid in the thermodynamic state points where gels were found. For the case of short-range attractive interactions, crystallization takes place on the time scale of the simulations if the quench into the metastable spinodal region is moderately deep. ${ }^{9,39} \mathrm{In}$
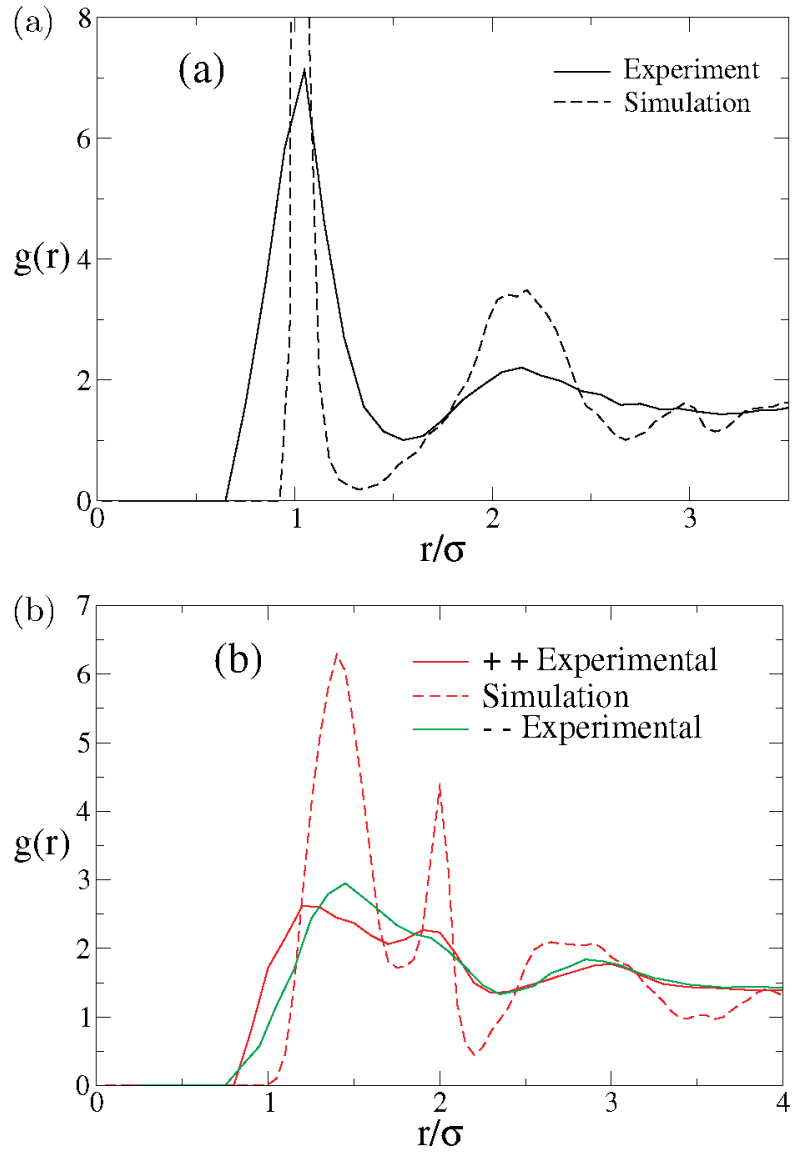

Figure 11. Comparison between the experimental and the simulated $g(r)$ (a) for oppositely charged and (b) for equally charged particles. The experimental $g(r)$ is obtained as an average over four samples. In the simulations, the $g(r)$ corresponds to a gel formed with $\kappa \sigma=2$ and $u^{*}=30 k_{\mathrm{B}} T, 945 \sigma^{2} /\left(D_{0} u^{*}\right)$ after the initial quench.

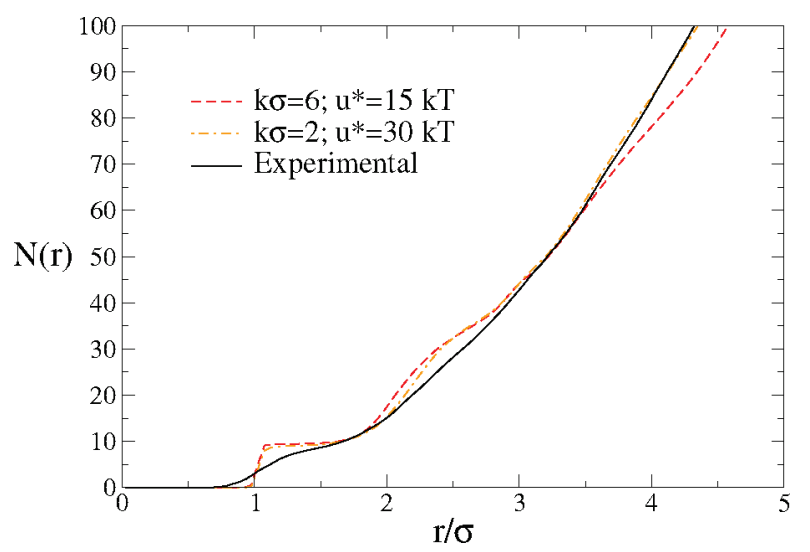

Figure 12. Comparison of $N(r)$, calculated for oppositely charged interactions, between experiments and simulations. In order to calculate the experimental $N(r)$, a thin slice of the gel where $\phi \approx$ 0.1 has been considered. The simulations correspond to gels formed $945 \sigma^{2} /\left(D_{0} u^{*}\right)$ after the quench with Yukawa parameters as indicated in the legend.

that case, a porous solid is formed due to crystallization of the liquid branches in the phase-separating system. In contrast, when the quench is sufficiently deep, no crystals are formed, because of vitrification prior to crystal nucleation..$^{9,39}$ In order to check whether there are crystalline domains present in our system, we have used a local bond order parameter. ${ }^{42,43}$ The idea is to calculate a vector $\mathbf{q}_{l}^{i}$ for each particle, whose components depend on the relative orientation with respect to the neighbouring 


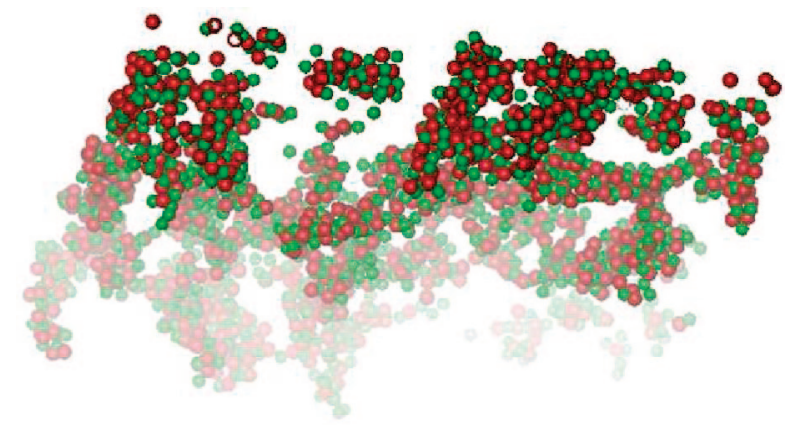

Figure 13. Rendering of the experimentally determined particle coordinates in gel "stalactites" hanging from the top of the capillary.
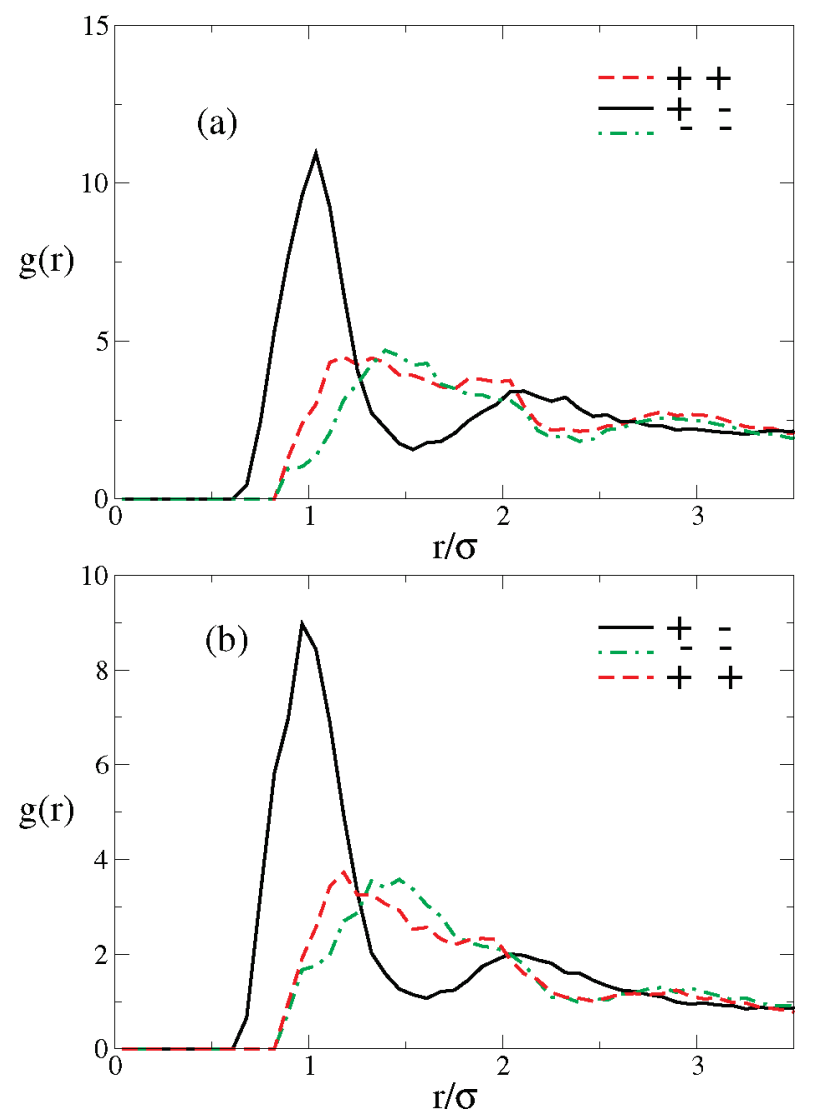

Figure 14. Comparison between the $g(r)$ of a gel sedimented on the bottom of the capillary (a) and another one hanging form the top of it (b).

particles. The component $m$ of the vector associated with the $i$ th particle is given by

$$
q_{l, m}^{i}=\frac{1}{N_{n}(i)} \frac{\sum_{j}^{N_{n}(i)} Y_{l m}\left(\mathbf{r}_{i j}\right)}{\left(\mathbf{q}_{l}^{i} \mathbf{q}_{l}^{i *}\right)^{1 / 2}}, \quad m=[-6,6]
$$

where, for our case, $l=6, N_{n}(i)$ is the number of particles closer than $1.33 \sigma$ to particle $i$, and $Y_{l, m}\left(r_{i j}\right)$ is a spherical harmonic function whose form depends on $l$ and $m$ and whose value depends on the relative orientation of particles $i$ and $j\left(r_{i j}\right)$. In a perfect body-centered cubic (bcc) crystal, all the particles have the same environment, and, therefore, the dot product between the vectors associated with any pair of particles is 1 . The dot product decreases when thermal vibrations are present, but, on average, it is closer to 1 if the central particles have a solid

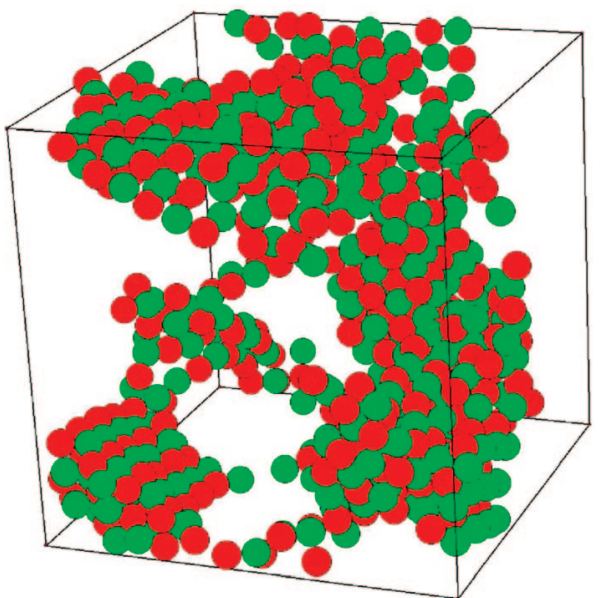

Figure 15. Structure formed $1190 \sigma^{2} /\left(u^{*} D_{0}\right)$ after the quench to $\kappa \sigma$ $=2$ and $u^{*}=20$. A solid domain is formed in the lower left corner of the simulation box. Same color particles have the same sign of charge.

rather than a liquid-like environment. Exploiting this, we are able to identify liquid and solid-like particles. We consider a particle as solid-like if at least $50 \%$ of the dot products with its neighbors is bigger than 0.5 . Any two solid-like particles that are closer than $1.3 \sigma$ are considered to belong to the same solid cluster. In this way we have checked that neither in the experiments nor in the simulations for the shorter ranged interactions $\left(\kappa \sigma=6 ; u^{*}=6.5,8,10\right.$, and 15$)$ are there solid domains present in the system, contrary to what happens for purely attractive short ranged interactions. ${ }^{9,39}$ The difference is probably related to the fact that the formation of an ordered binary crystal of oppositely charged particles can be kinetically hindered by the slow incorporation of particles into a growing crystal nucleus. ${ }^{43}$ Nevertheless, when the range of interaction is longer $(\kappa \sigma=2)$, particles can rearrange more easily, therefore facilitating the formation of solid domains inside the network of particles. Consequently, for $\kappa \sigma=2$, we have detected a crystalline domain of significant size (more than 100 particles) for the simulations corresponding to $u^{*}=20$ (see Figure 15). For deeper quenches $\left(u^{*}=25\right.$ or $\left.u^{*}=30\right)$, no crystallization has been seen, since, at large energies at contact, rearrangements towards the crystalline state are too slow. For $\kappa \sigma=2$ and a more shallow quench $\left(u^{*}=15\right)$, we have not detected any solid cluster either, given that the nucleation barrier for this energy at contact is larger than for $u^{*}=20$. Thus, our simulations suggest that crystallization is more likely to happen for longrange interactions and quenches moderately deep into the metastable spinodal region.

The absence (or presence) of crystallization is related to the mechanical strength of the gel-like structures. If particle rearrangements are slow, the gel structure will be rigid and crystallization will be hindered. On the other hand, if rearrangements are easier, this means faster network collapse and more crystallization. This explains why depletion gels collapse $\mathrm{s}^{44,41,40}$ and crystallize ${ }^{39}$ more readily than gels of oppositely charged particles. Namely, when a charged particle tries to escape from its "cage", formed by oppositely charged particles, it will most likely encounter a like-charged particle in the neighbouring cage; this hinders the re-arrangement. For particles in a depletion gel, this effect does not occur, because they all attract each other, making rearrangements easier.

\section{Conclusions}

In summary, we have studied, both experimentally and by computer simulations, the formation of dynamically arrested 
structures at low densities in a system of oppositely charged colloids. We have shown in detail how the system evolves from a low-density homogeneous fluid to a non-ergodic gel. The mechanism observed is typical of an interrupted spinodal decomposition: initially, the system forms a network of thin branches, which then thickens and coarsens until the percolating network evolves very slowly. Using BD computer simulations, we have compared the structural evolution of homogeneous suspensions destined to form a gel with that of a plain spinodal decomposition. We can conclude that, for the parameters studied, the mechanism of formation of low-density dynamically arrested states for this system is an interrupted gas-liquid spinodal decomposition. This is also the case for purely attractive systems with short-ranged isotropic interactions. ${ }^{7,8}$ Note, however, that according to theoretical predictions it should be possible to obtain gels at supercritical temperatures (independently of the gas-liquid phase separation). ${ }^{13}$ Nevertheless, this has to be further explored, since gels at supercritical temperatures have not been found yet either in experiments or in simulations, neither for purely attractive nor for oppositely charged particles. Likewise, it would be interesting to check in future work whether gel formation can be seen as an arrested gas-liquid phase separation in the limit of very strong interactions (low temperatures), where every collision gives rise to an irreversible bond and the assumption of the presence of locally defined thermodynamic properties breaks down.

We have studied two different interaction ranges by means of BD simulations. The shorter corresponds to a phase diagram in which the critical point is metastable, whereas the longerranged interaction leads to a phase diagram with a stable gas-liquid phase separation. Gels are formed for both interaction ranges, provided that the system is quenched well inside a region of the phase diagram where the gas-liquid coexistence is metastable. Since almost all the gel-forming systems studied so far are based on short-ranged interacting potentials, we want to stress that, by increasing the interaction strength, it is possible to form gels for long-ranged interactions.

We have also checked that the structure of the experimental and simulated gels is similar. This suggests that effects such us hydrodynamic interactions or gravity do not substantially affect the local structure of gels of oppositely charged particles. Additionally, the structure of gels stretched by gravity is similar to that of gels squeezed by gravity, which corroborates that the effect of gravity in this type of gels is not crucial. The long persistence of the gels is extra proof for that.

Finally, we have analyzed whether crystalline domains were formed in a system quenched to a state point where the gas-liquid coexistence is metastable. From our preliminary analysis we can conclude that crystallization is less likely for oppositely charged colloids than for purely attractive systems. In our system, crystallization is more favored for moderate quenches and long-ranged interactions.

In conclusion, both for systems of oppositely charged colloids and for colloid-polymer mixtures, gels can be formed by quenching to a point in the phase diagram where the gas-liquid coexistence is metastable. Thus, the equilibrium phase diagram tells us what combination of interaction parameters can cause the arrest of the system in an out-ofequilibrium state. At the same time, however, it appears that gels of oppositely charged colloids do not collapse nor crystallize as readily as gels formed in colloid-polymer mixtures. In future investigations, it will be of great interest to study the relation between the interaction characteristics and the mechanical properties in more detail.
Acknowledgment. E.S. warmly thanks useful discussions with A. Puertas, F. Sciortino, and E. Zaccarelli in the Soft Matter International Meeting held in Aachen in October 2007, and C. Valeriani for bringing the authors together in the lab with her enthusiasm. This work was financially supported by the Nederlandse Organisatie voor Wetenschappelijk Onderzoek (NWO). This work was supported by the Stichting voor Fundamenteel Onderzoek der Materie, the Nederlandse Organisatie voor Wetenschappelijk Onderzoek.

Supporting Information Available: We provide, as additional material, movies showing the whole sequence of frames corresponding to Figure 3. The movies illustrate the formation of a gel starting from a dilute suspension. The first file corresponds to Figure 3a (solvent flow), whereas the second file corresponds to Figure $3 b$ (no solvent flow). This material is available free of charge via the Internet at http:// pubs.acs.org.

\section{References and Notes}

(1) Avnir, D., Ed. The Fractal Approach to Heterogeneous Chemistry: Surfaces, Colloids, Polymers; John Wiley \& Sons: New York, 1989.

(2) Barabasi, A.; Stanley, H. E. Fractal Concepts in Surface Growth; Cambridge University Press: Cambridge, U.K., 1995.

(3) Poon, W. C. K.; Haw, M. D. Adv. Colloid Interface Sci. 1997, 73, 71.

(4) Witten, T. A.; Sander, L. M. Phys. Rev. Lett. 1981, 47, 1400.

(5) Manley, S.; Wyss, H. M.; Miyazaki, K.; Conrad, J. C.; Trappe, V.; Kaufman, L. J.; Reichman, D. R.; Weitz, D. A. Phys. Rev. Lett. 2005, 95, 238202.

(6) Foffi, G.; Michele, C. D.; Sciortino, F.; Tartaglia, P. J. Chem. Phys. 2005, 122, 224903.

(7) Buzzaccaro, S.; Rusconi, R.; Piazza, R. Phys. Rev. Lett. 2007, 99, 098301.

(8) Lu, P. J.; Zaccarelli, E.; Ciulla, F.; Schofield, A. B.; Sciortino, F.; Weitz, D. Nature 2008, 453, 06931.

(9) Charbonneau, P.; Reichman, D. R. Phys. Rev. E 2007, 75, 011507.

(10) Zaccarelli, E.; Buldyrev, S.; Nave, E. L.; Moreno, A. J.; SaikaVoivod, I.; Sciortino, F.; Tartaglia, P. Phys. Rev. Lett. 2005, 94, 218301.

(11) Zaccarelli, E.; Saika-Voivod, I.; Buldyrev, S. V.; Moreno, A. J.; Tartaglia, P.; Sciortino, F J. Chem. Phys 2006, 124, 124908.

(12) Zaccarelli, E. J. Phys.: Condens. Matter 2007, 19, 323101.

(13) Bergenholtz, J.; Poon, W. C. K.; Fuchs, M Langmuir 2003, 19, 4493.

(14) Kroy, K.; Cates, M. E.; Poon, W. C. K. Phys. Rev. Lett. 2004, 92, 148302.

(15) Pham, K. N.; Puertas, A. M.; Bergenholtz, J.; Egelhaaf, S. U.; Moussaid, A.; Pusey, P. N.; Schofield, A. B.; Cates, M. E.; Fuchs, M.; Poon, W. C. K. Science 2002, 296, 104.

(16) van Blaaderen, A. Prog. Colloid Interface Sci. 1997, 104, 59.

(17) Campbell, A. I.; Anderson, V. J.; van Duijneveldt, J. S.; Bartlett, P. Phys. Rev. Lett. 2005, 94, 208301.

(18) Dibble, C. J.; Kogan, M.; Solomon, M. J. Phys. Rev. E 2006, 74, 041403.

(19) Lu, P. J.; Conrad, J. C.; Wyss, H. M.; Schofield, A. B.; Weitz, D. A. Phys. Rev. Lett. 2006, 96, 028306.

(20) Lodge, J. F. M.; Heyes, D. M. Phys. Chem. Chem. Phys. 1999, 1, 2119.

(21) Del Gado, E.; Kob, W. Phys. Rev. Lett. 2007, 98, 028303.

(22) Piazza, R.; Di Pietro, G. Europhys. Lett. 1994, 28, 445.

(23) Cates, M. E.; Fuchs, M.; Kroy, K.; Poon, W. C. K.; Puertas, A. M. J. Phys.: Condens. Matter 2004, 16, S4861.

(24) Kim, A. Y.; Hauch, K. D.; Berg, J. C.; Martin, J. E.; Anderson, R. A. J. Colloid Interface Sci. 2003, 260, 149.

(25) Romero-Cano, M. S.; Caballero, J. B.; Puertas, A. M. J. Phys. Chem. B 2006, 110, 13220 .

(26) Caballero, J. B.; Puertas, A. M. Phys. Rev. E 2007, 76, 011401.

(27) Leunissen, M. E.; Christova, C. G.; Hynninen, A. P.; Royall, C. P.; Campbell, A. I.; Imhof, A.; Dijkstra, M.; van Roij, R.; van Blaaderen, A. Nature 2005, 437, 235.

(28) Hynninen, A.-P.; Leunissen, M. E.; van Blaaderen, A.; Dijkstra, M. Phys. Rev. Lett. 2006, 96, 018303.

(29) Hynninen, A.-P.; Christova, C.; van Roij, R.; van Blaaderen, A.; Dijkstra, M. Phys. Rev. Lett. 2006, 96, 138308.

(30) Fortini, A.; Hynninen, A.; Dijkstra, M. J. Chem. Phys. 2006, 125, 094502. 
(31) Bosma, G.; Pathmamanoharan, C.; de Hoog, E.; Kegel, W.; van Blaaderen, A.; Lekkerkerker, H. J. Colloid Interface Sci. 2002, 245, 292. (32) Royall, C.; Leunissen, M.; van Blaaderen, A. J. Phys.: Condens. Matter 2003, 15, S3581.

(33) Leunissen, M. E. Manipulating colloids with charges and electric fields. Ph.D. Thesis, Utrecht University, 2007, available at www.colloid.nl. (34) Yamamoto, R.; Kim, K.; Nakayama, Y.; Miyazaki, K.; Reichman, D. arXiv:cond-mat/0604404v1 2006.

(35) Charbonneau, P.; Reichman, D. R. Phys. Rev. E 2007, 75, 011507.

(36) Caballero, J. B.; Noya, E. G.; Vega, C. J. Chem. Phys. 2007, 127, 244910

(37) Sciortino, F.; Tartaglia, P.; Zaccarelli, E. J. Phys. Chem. B 2005, 109, 21942.

(38) Blaak, R.; Miller, M. A.; Hansen, J.-P. Europhys. Lett. 2007, 78, 26002 .
(39) Fortini, A. Bulk and confinement-induced phase transitions in colloidal suspensions. Ph.D. Thesis, Utrecht University, 2007, available at www.colloid.nl.

(40) d'Arjuzon, R. J. M.; Frith, W.; Melrose, J. R. Phys. Rev. E 2003, $67,061404$.

(41) Starrs, L.; W, C. K; Poon, D. J. H.; Robins, M. M J. Phys.: Condens. Matter 2002, 14, 2485.

(42) ten Wolde, P. R.; Ruiz-Montero, M. J.; Frenkel, D. J. Chem. Phys. 1996, 104, 9932.

(43) Sanz, E.; Valeriani, C.; Frenkel, D.; Dijkstra, M. Phys. Rev. Lett. 2007, 99, 55501 .

(44) de Hoog, E. H. A.; Kegel, W. K.; van Blaaderen, A.; Lekkerkerker, H. N. W. Phys. Rev. E 2001, 64, 021407.

JP801440V 